\title{
Correction: Berberine-targeted miR-21 chemosensitizes oral carcinomas stem cells
}

\section{Che-Yi Lin ${ }^{1}$, Pei-Ling Hsieh ${ }^{2, *}$, Yi-Wen Liao ${ }^{3, *}$, Chih-Yu Peng ${ }^{3}$, Ming-Yi Lu ${ }^{3}$, Ching- Hsuan Yang ${ }^{2}$, Cheng-Chia Yu ${ }^{2,3,4}$ and Chia-Ming Liu ${ }^{3,4}$}

1 Department of Oral and Maxillofacial Surgery, Chi Mei Hospital, Tainan, Taiwan

2 Institute of Oral Sciences, Chung Shan Medical University, Taichung, Taiwan

${ }^{3}$ School of Dentistry, Chung Shan Medical University, Taichung, Taiwan

${ }^{4}$ Department of Dentistry, Chung Shan Medical University Hospital, Taichung, Taiwan

* These authors have contributed equally to this work

Published: May 15, 2018

Copyright: Lin et al. This is an open-access article distributed under the terms of the Creative Commons Attribution License 3.0 (CC BY 3.0), which permits unrestricted use, distribution, and reproduction in any medium, provided the original author and source are credited.

This article has been corrected: The correct 4th affiliation is given below:

${ }^{4}$ Department of Dentistry, Chung Shan Medical University Hospital, Taichung, Taiwan

Original article: Oncotarget. 2017; 8:80900-80908. https://doi.org/10.18632/oncotarget.20723 